\title{
Receiver Multiuser Diversity Aided Multi-Stage MMSE Multiuser Detection: A Low-Complexity Detector Fast-Converging to the Optimum
}

\author{
Lie-Liang Yang \\ School of ECS, University of Southampton, SO17 1BJ, United Kingdom \\ Tel: 0044-(0)23-8059 3364, Email: 1ly@ecs.soton.ac.uk, http://www-mobile.ecs.soton.ac.uk
}

\begin{abstract}
Design of low-complexity detection schemes that can approach near-optimum bit error rate (BER) performance in multiuser or other multiple-input multiple-output (MIMO) systems has always been highly challenging. In this paper we propose and investigate a so-called receiver multiuser diversity aided multi-stage minimum mean-square error multiuser detection (RMD/MS-MMSE MUD) scheme operated in the principles of successive interference cancellation (SIC). The BER performance of the RMD/MS-MMSE MUD is investigated in association with both the direct-sequence code-division multiple-access (DS-CDMA) communicating over both Gaussian and Rayleigh fading channels, and the spacedivision multiple-access (SDMA) communicating over Rayleigh fading channels. Furthermore, we assume that the DS-CDMA and SDMA systems are either full-load or overload. Our studies show that the RMD/MSMMSE MUD in full-load cases is capable of converging to the optimum BER performance of the maximum likelihood (ML) multiuser detector (MUD). For the overload systems, the RMD/MS-MMSE MUD can make a DS-CDMA or SDMA system support $K=2 N$ users, but still achieve much better BER performance than a corresponding DS-CDMA or SDMA system using the conventional MMSE-MUD to support $K=N$ users, where $N$ denotes the spreading factor of DS-CDMA or the number of receive antennas of SDMA.
\end{abstract}

\section{INTRODUCTION}

In multiuser detection the optimum maximum a-posteriori (MAP) and ML MUDs [1-3] are capable of achieving the optimum BER performance that is close to the single-user BER bound. However, the complexity of these optimum MUDs is exponentially proportional to the number of users supported, which becomes extreme even when a moderate number of users are considered. Consequently, the application of the optimum MUDs in practice is limited. For this sake, research efforts have been put on finding the suboptimum solutions with practical complexity for the MAP or ML MUD problems, since the invention of the optimum MUDs [1,2]. In general, the suboptimum MUDs towards the optimum MUD problems can be divided into two classes. The first class aim at designing efficient search or non-search algorithms, such as those summarized in [4] and [5], in order to find approximate solutions for the optimum MUDs. Despite significant complexity reduction, however, these suboptimum algorithms either are still too complex to be implemented or achieve much worse BER performance than the optimum MUDs [5].

The second class of suboptimum MUDs do not solve directly the optimum MUD problems. Instead, they approximate the optimum MUD problems by some suboptimum MUD problems that can be solved with lower complexity. This second class of suboptimum MUDs include typically the various types of decision feedback or interference cancellation (IC) assisted MUDs, such as those studied in [4, 6-9]. The BER performance of the conventional IC-aided MUDs is usually much worse than that of the optimum MUDs, as shown, e.g., in $[3,4,6]$, due to the severe error propagation. In comparison with the conventional IC-based MUDs, the IC-based V-BLAST systems [7-9] are capable of achieving much better BER performance. However, as the studies in [10] shown, the V-BLAST schemes become less efficient as the system size increases, where the system size is reflected by the spreading factor $N$ of DS-CDMA systems, the number of receive antennas $N$ of SDMA systems, etc. Furthermore, the studies in the context of the V-BLAST systems have so far been constrained mainly on the under-load or full-load multiantenna systems with the number of transmit antennas not exceeding the number of receive antennas.

In this paper we propose and investigate a so-called RMD/MSMMSE MUD, which belongs to the family of SIC operated in the principles similar to that of the V-BLAST [7-9]. We motivate to reveal the fact that a low-complexity SIC scheme is capable of achieving the BER performance approaching that of the optimum MUDs, provided that the following two conditions are satisfied. First, the reliabilities of different users can be measured in an optimum way near independently; the more independent the measurements are, the closer the BER is to that of the optimum MUDs. Second, the realtime reliabilities are highly sparse, revealing that some users are much more reliable than some others. Fortunately, as shown in this contribution, the above-mentioned two conditions in the multiuser systems using MMSE-MUD can usually be satisfied approximately, especially when relatively large multiuser systems are considered, or if noise dominates the achievable BER performance. Therefore, in this paper we first introduce a concept of RMD, in order to illustrate the sparsity of reliabilities in multiuser systems. Our studies show that, when measured in MAP sense, the reliabilities of different users are usually highly diverse, when communicating over either Gaussian or Rayleigh fading channels. Hence, a MAP-based reliability measurement scheme is then proposed for ordering the users to be detected by the RMD/MS-MMSE MUD. The proposed reliability measurement scheme can jointly take account of the effects from fading and realtime Gaussian noise, and it converges to the optimum as the size of the multiuser systems increases. Finally, in this contribution we investigate the BER performance of the RMD/MS-MMSE MUD in the context of both the DS-CDMA and SDMA systems, when they are either fullload or overload. Our studies and performance results show that the RMD/MS-MMSE MUD converges the optimum as the size of the DSCDMA or SDMA systems increases. It is high-efficiency even when the DS-CDMA or SDMA systems are heavily loaded. For example, even supporting $K=2 N$ number of users, the DS-CDMA or SDMA systems using the RMD/MS-MMSE MUD still achieve better BER performance than the corresponding DS-CDMA or SDMA systems using the conventional MMSE-MUD to support only $K=N$ users.

\section{Principles of Receiver Multiuser Diversity}

Consider a base-station (BS) detecting simultaneously $K$ number of binary phase-shift keying (BPSK) user signals based on the decision variables

$$
y_{k}=b_{k}+n_{k}, k=1,2, \ldots, K
$$

where $b_{k} \in\{+1,-1\}$ is assumed to be an independently identically distributed (iid) random variable with $P\left(b_{k}=+1\right)=P\left(b_{k}=-1\right)=$ 0.5 and $n_{k}$ obeys Gaussian distribution with mean zero and a variance of $\sigma^{2}=1 / 2 \gamma_{k}$, where $\gamma_{k}=\alpha_{k}^{2} \gamma_{b}$ denotes the instantaneous signalto-noise ratio (SNR) per bit, while $\gamma_{b}$ denotes the average SNR per bit. Furthermore, $\alpha_{k}=1$ corresponds to the Gaussian channel scenarios, while $\alpha_{k}$ obeys the iid Rayleigh distribution associated with $E\left[\alpha_{k}^{2}\right]=$ 1 when communicating over Rayleigh fading channels. 
Below we investigate the reliabilities for detection of $\left\{b_{k}\right\}$ and the BER performance of the bits at the $K$ different reliability levels, without minding which bit is received from which user. Our results show that the BER performance corresponding to the $K$ different reliability levels is highly diverse, when communicating over either Gaussian or Rayleigh fading channels. Hence, we refer to this type of diversity as the receiver multiuser diversity (RMD), since the diversity is provided by multiusers. Furthermore, 'receiver' is explicitly used to indicate that the diversity does not invoke any assistance from the transmitters.

The reliability of detecting $b_{k}$ can be measured in an optimum way by the magnitude of the posteriori log-likelihood ratio (LLR) [11], which, when $b_{k}$ is an iid random variable, is expressed as

$$
L_{k}=\left|\ln \left[\frac{f\left(b_{k}=+1 \mid y_{k}\right)}{f\left(b_{k}=-1 \mid y_{k}\right)}\right]\right|=\left|\ln \left[\frac{f\left(y_{k} \mid b_{k}=+1\right)}{f\left(y_{k} \mid b_{k}=-1\right)}\right]\right|
$$

Applying the Gaussian probability density function (PDF) of $y_{k}$ into the above equation yields

$$
L_{k}=\left|4 \gamma_{k} y_{k}\right| \triangleq \gamma_{k}\left|y_{k}\right|, k=1,2, \ldots, K
$$

Let us order in ascent the reliabilities of $K$ bits as

$$
L_{(1)} \leq L_{(2)} \leq \cdots \leq L_{(K)}
$$

where $L_{(i)}, i=1, \ldots, K$, represents the reliability of the $i$ th most unreliable in $\left\{L_{1}, L_{2}, \ldots, L_{K}\right\}, L_{(1)}$ and $L_{(K)}$ correspond to the most unreliable bit and the most reliable bit, respectively.

After some derivations, it can be shown that the BER of the bits at the $i$ th reliability level for communications over Gaussian channels can be expressed as

$$
\begin{aligned}
P_{E}(i)= & K\left(\begin{array}{c}
K-1 \\
i-1
\end{array}\right) \frac{1}{\sqrt{2 \pi}} \int_{\sqrt{2 \gamma_{b}}}^{\infty} \exp \left(-\frac{t^{2}}{2}\right) \\
& \times\left[1-Q(t)-Q\left(t-2 \sqrt{2 \gamma_{b}}\right)\right]^{i-1} \\
& \times\left[Q(t)+Q\left(t-2 \sqrt{2 \gamma_{b}}\right)\right]^{K-i} d t \\
& i=1,2, \ldots, K
\end{aligned}
$$

By contrast, when communicating over Rayleigh fading channels, it can be shown that the BER of the bits at the $i$ th reliability level can be formulated as

$$
\begin{aligned}
P_{E}(i)= & \sum_{m=0}^{i-1} \sum_{n=0}^{K-i+m}(-1)^{m} K\left(\begin{array}{c}
K-1 \\
i-1
\end{array}\right)\left(\begin{array}{c}
i-1 \\
m
\end{array}\right)\left(\begin{array}{c}
K-i+m \\
n
\end{array}\right) \\
& \times \frac{D A^{n} B^{K-i+m-n}}{n+1-(K-i+m-n) C}, i=1, \ldots, K
\end{aligned}
$$

where, by definition,

$$
\begin{aligned}
A & =\frac{1}{2}\left(1-\sqrt{\frac{\gamma_{b}}{1+\gamma_{b}}}\right), B=\frac{1}{2}\left(1+\sqrt{\frac{\gamma_{b}}{1+\gamma_{b}}}\right) \\
C & =2 \sqrt{\gamma_{b}\left(1+\gamma_{b}\right)}-2 \gamma_{b}-1, \\
D & =\frac{1}{2\left(\sqrt{\gamma_{b}\left(1+\gamma_{b}\right)}+\gamma_{b}+1\right)}
\end{aligned}
$$

Figs. 1 and 2 illustrate the BER evaluated by (5) and (6) for the case of $K=8$. In both figures the conventional BPSK scheme's BER, which is in fact the average BER or unordered BER, is also depicted. Furthermore, in Fig. 2 considering the Rayleigh fading channels, the BER of the BPSK communicating over Gaussian channels is also provided. From the results of Figs. 1 and 2 we can observe that the BER at different reliability levels is highly diverse for communications over either Gaussian or Rayleigh fading channels. This observation implies that RMD exists in both Gaussian and fading channels, in contrast to the other type of diversity, such as frequency-diversity,

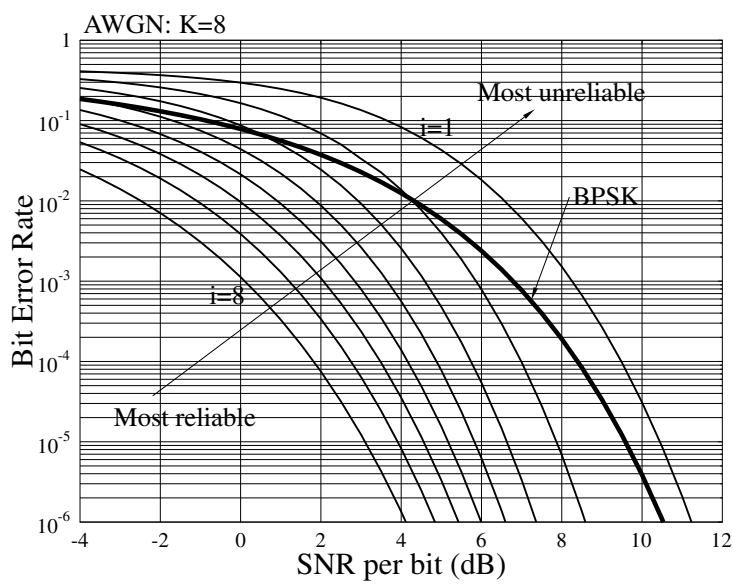

Fig. 1. BER versus SNR per bit $\left(\gamma_{b}\right)$ for the bits at different reliability levels when communicating over Gaussian channels.

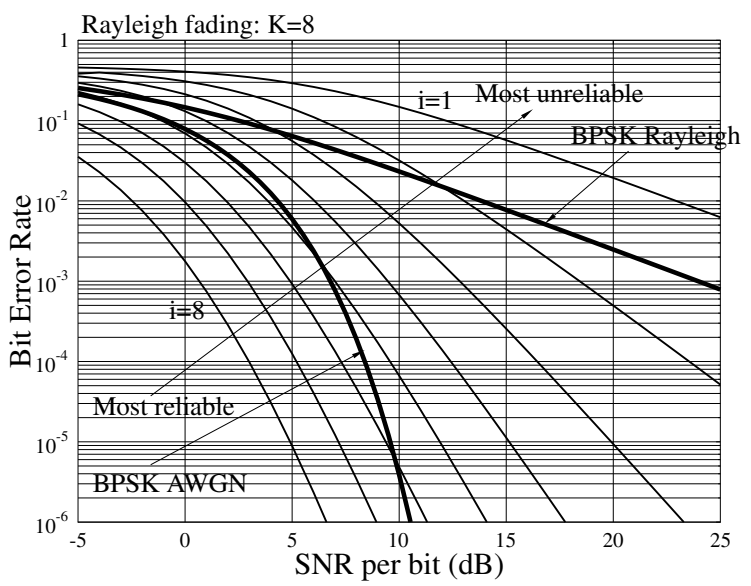

Fig. 2. BER versus average SNR per bit $\left(\gamma_{b}\right)$ for the bits at different reliability levels when communicating over Rayleigh fading channels.

spatial-diversity, etc., which only exist when communicating over fading channels. As shown in Figs. 1 and 2, the average BER, i.e, the BER of BPSK, is generally dominated by the several most unreliable bits, and is mainly dominated by the two most unreliable bits, provided that the SNR $\gamma_{b}$ is sufficiently high. Furthermore, when communicating over Rayleigh fading channels, as seen in Fig. 2, the BER of the bits at relatively high reliability levels can be lower than the BER of the BPSK over Gaussian channels, if the value of $K$ is sufficiently high.

\section{MMSE Detection and Reliability MEasurement}

\section{A. MIMO Model and MMSE Detection}

In both DS-CDMA and SDMA systems using BPSK baseband modulation and supporting $K$ number of synchronous users, the MIMO model describing the output-input relationship can be formulated by the MIMO equation as

$$
\boldsymbol{y}=\boldsymbol{H b}+\boldsymbol{n}
$$

where $\boldsymbol{y}$ and $\boldsymbol{n}$ are $N$-length complex-valued observation vector and noise vector, $\boldsymbol{b}=\left[b_{1}, b_{2}, \cdots, b_{K}\right]^{T}$ contains the binary bits transmitted by the $K$ users and $\boldsymbol{H}=\left[\boldsymbol{h}_{1}, \boldsymbol{h}_{2}, \cdots, \boldsymbol{h}_{K}\right]$ is an $(N \times K)$ matrix. For DS-CDMA systems, the matrix $\boldsymbol{H}$ can be further written as $\boldsymbol{H}=$ 
$\boldsymbol{C A}$, where $\boldsymbol{C}$ is the $(N \times K)$ spreading matrix with the $k$ th column representing the $k$ th user's DS spreading sequence, while the matrix $\boldsymbol{A}=\operatorname{diag}\left\{a_{1}, a_{2}, \cdots, a_{K}\right\}$ is an $(K \times K)$ diagonal matrix, where $a_{k}=e^{j \theta_{k}}$ for Gaussian channels and obeys iid complex Gaussian distribution with mean zero and $E\left[\left|a_{k}\right|^{2}\right]=1$ for Rayleigh fading channels. For both DS-CDMA and SDMA, we assume that the matrix $\boldsymbol{H}$ is normalized to $E\left[\boldsymbol{H}^{H} \boldsymbol{H}\right]=\boldsymbol{I}$. Correspondingly, we assume that $\boldsymbol{n}$ is a multivariate complex-valued Gaussian noise vector distributed with mean zero and a covariance matrix $E\left[\boldsymbol{n} \boldsymbol{n}^{H}\right]=2 \sigma^{2} \boldsymbol{I}_{N}$. For the DS-CDMA, $\sigma^{2}=1 /(2 \gamma)$ with $\gamma$ representing the average signal-tonoise ratio (SNR) per symbol. By contrast, for the SDMA, we have $\sigma^{2}=1 /(2 N \gamma)$, where $\gamma$ denotes the average SNR per symbol per receive antenna.

In this contribution we explore mainly the achievable BER performance of the DS-CDMA and SDMA systems using RMD/MS-MMSE MUD, when these systems are heavily loaded. A system is referred to as a full-load system when $K=N$ and as an overload system if $K>N$

When MMSE-MUD is employed, the decision variables for the $K$ users can be expressed as

$$
\boldsymbol{z}=\Re\left\{\boldsymbol{W}^{H} \boldsymbol{y}\right\}, \text { or } z_{k}=\Re\left\{\boldsymbol{w}_{k}^{H} \boldsymbol{y}\right\}, k=1,2, \ldots, K
$$

where $\boldsymbol{z}=\left[z_{1}, z_{2}, \cdots, z_{K}\right]^{T}$ is an $K$-length decision variable vector, $\boldsymbol{W}=\left[\boldsymbol{w}_{1}, \boldsymbol{w}_{2}, \ldots, \boldsymbol{w}_{K}\right]$ is an $(N \times K)$ weight matrix, while $\boldsymbol{w}_{k}$ is an $N$-length weight vector. When the MMSE-MUD is employed, we have $[3,4]$,

$$
\boldsymbol{W}=\boldsymbol{R}_{y}^{-1} \boldsymbol{H}, \text { or } \boldsymbol{w}_{k}=\frac{\boldsymbol{R}_{k}^{-1} \boldsymbol{h}_{k}}{1+\boldsymbol{h}_{k}^{H} \boldsymbol{R}_{k}^{-1} \boldsymbol{h}_{k}}
$$

where $\boldsymbol{R}_{y}$ and $\boldsymbol{R}_{k}$ denote, respectively, the autocorrelation matrix of $y$ and the autocorrelation matrix of the interference-plus-noise, which are given by

$$
\begin{aligned}
& \boldsymbol{R}_{y}=\boldsymbol{H} \boldsymbol{H}^{H}+\sigma^{2} \boldsymbol{I}_{N}=\sum_{l=1}^{K} \boldsymbol{h}_{l} \boldsymbol{h}_{l}^{H}+\sigma^{2} \boldsymbol{I}_{N} \\
& \boldsymbol{R}_{k}=\sum_{l \neq k}^{K} \boldsymbol{h}_{l} \boldsymbol{h}_{l}^{H}+\sigma^{2} \boldsymbol{I}_{N}=\boldsymbol{R}_{y}-\boldsymbol{h}_{k} \boldsymbol{h}_{k}^{H}
\end{aligned}
$$

Upon substituting (8) and $\boldsymbol{w}_{k}$ from (10) into (9), one can express $z_{k}$ as

$$
z_{k}=\frac{\boldsymbol{h}_{k}^{H} \boldsymbol{R}_{k}^{-1} \boldsymbol{h}_{k}}{1+\boldsymbol{h}_{k}^{H} \boldsymbol{R}_{k}^{-1} \boldsymbol{h}_{k}} b_{k}+\Re\left\{\frac{\boldsymbol{h}_{k}^{H} \boldsymbol{R}_{k}^{-1}}{1+\boldsymbol{h}_{k}^{H} \boldsymbol{R}_{k}^{-1} \boldsymbol{h}_{k}}\left(\boldsymbol{H} \boldsymbol{b}-\boldsymbol{h}_{k} b_{k}+\boldsymbol{n}\right)\right\},
$$

which according to [12] can be closely approximated as a Gaussian random variable with its PDF given by

$$
f\left(z_{k} \mid b_{k}\right)=\frac{1}{\sqrt{2 \pi} \sigma_{k}} \exp \left[-\frac{\left(z_{k}-m_{k}\right)^{2}}{2 \sigma_{k}^{2}}\right]
$$

associated with the mean and variance given by

$$
m_{k}=\frac{\bar{\gamma}_{k}}{1+\bar{\gamma}_{k}} b_{k}, \quad \sigma_{k}^{2}=\frac{\bar{\gamma}_{k}}{2\left(1+\bar{\gamma}_{k}\right)^{2}}
$$

In (15) $\bar{\gamma}_{k}=\boldsymbol{h}_{k}^{H} \boldsymbol{R}_{k}^{-1} \boldsymbol{h}_{k}$ represents the instantaneous signal-tointerference-plus-noise ratio (SINR) for detection of user $k$. Furthermore, the decision variables $z_{1}, z_{2}, \cdots, z_{K}$ become more independent, as the value of $N$ increases.

\section{B. Reliability Measurement in MMSE Detection}

Given the decision variable vector $\boldsymbol{z}$ as shown in (9) for the MMSEMUD, the reliability of detecting $b_{k}$ can be measured in an optimum way by the magnitude of the posteriori LLR [11]

$$
\begin{aligned}
L_{k} & =\left|\ln \left[\frac{f\left(b_{k}=+1 \mid \boldsymbol{z}\right)}{f\left(b_{k}=-1 \mid \boldsymbol{z}\right)}\right]\right|=\left|\ln \left[\frac{f\left(\boldsymbol{z}, b_{k}=+1\right)}{f\left(\boldsymbol{z}, b_{k}=-1\right)}\right]\right| \\
& =\left|\ln \left[\frac{\sum_{\boldsymbol{b}_{k}^{+} \in\{+1,-1\}^{K}} f\left(\boldsymbol{z}, \boldsymbol{b}_{k}^{+}\right)}{\sum_{\boldsymbol{b}_{k}^{-} \in\{+1,-1\}^{K}} f\left(\boldsymbol{z}, \boldsymbol{b}_{k}^{-}\right)}\right]\right|, k=1, \ldots, K
\end{aligned}
$$

where $\boldsymbol{b}_{k}^{+}$and $\boldsymbol{b}_{k}^{-}$are $K$-length binary vectors with their $k$ th entries being +1 and -1 , respectively.

From (16) and the principles of the MAP algorithm [11], we can have the following two observations. First, if $\boldsymbol{z}$ can be divided into two independent vectors $\boldsymbol{z}_{a}$ related to $K_{1}$ users and $\boldsymbol{z}_{b}$ related to the rest ( $K-K_{1}$ ) users, then, when assuming that $b_{k}$ is associated with $\boldsymbol{z}_{a}$, the reliability of detecting $b_{k}$ can be evaluated as

$$
L_{k}=\left|\ln \left[\frac{\sum_{\boldsymbol{b}_{k}^{+} \in\{+1,-1\}^{K}} f\left(\boldsymbol{z}_{a}, \boldsymbol{b}_{k}^{+}\right)}{\sum_{\boldsymbol{b}_{k}^{-} \in\{+1,-1\}^{K_{1}}} f\left(\boldsymbol{z}_{a}, \boldsymbol{b}_{k}^{-}\right)}\right]\right|
$$

which explains that the detection of $b_{k}$ or the reliability measurement of detecting $b_{k}$ is optimum without considering the bits in $z_{b}$. Hence, the reliabilities of the users with independent sets of decision variables can be measured independently. Second, if the $m$ th, $m \neq k$, user has been correctly detected, then (16) is reduced to

$$
L_{k}=\left|\ln \left[\frac{\sum_{\boldsymbol{b}_{k, m}^{+} \in\{+1,-1\}^{K}} f\left(\boldsymbol{z}, \boldsymbol{b}_{k, m}^{+}\right)}{\sum_{\boldsymbol{b}_{k, m}^{-} \in\{+1,-1\}^{K}} f\left(\boldsymbol{z}, \boldsymbol{b}_{k, m}^{-}\right)}\right]\right|, k \neq m
$$

where $\boldsymbol{b}_{k, m}^{+}$and $\boldsymbol{b}_{k, m}^{-}$are $K$-length binary vectors with their $k$ th entries being +1 and -1 , while with their $m$ th entries replaced by $\hat{b}_{m}$ of the estimate to $b_{m}$. Equation (18) implies that, once a user has been correctly detected, then cancellation of this detected user is optimum to the other not yet detected users.

Explicitly, the complexity for finding the optimum reliability $L_{k}$ based on (16) is $\mathcal{O}\left(2^{K}\right)$, which is extreme when the value of $K$ is high. Hence, according to (17), we approximate the decision variables $z_{1}, z_{2}, \ldots, z_{K}$ in $\boldsymbol{z}$ as iid random variables obeying the Gaussian distribution as shown in (14). In this case, by assuming $P\left(b_{k}=+1\right)=$ $P\left(b_{k}=-1\right)$, the reliability of (16) can be simplified to

$$
L_{k}=\left|\ln \left[\frac{f\left(z_{k}, b_{k}=+1\right)}{f\left(z_{k}, b_{k}=-1\right)}\right]\right|=\left|\ln \left[\frac{f\left(z_{k} \mid b_{k}=+1\right)}{f\left(z_{k} \mid b_{k}=-1\right)}\right]\right|
$$

Furthermore, when applying the PDF of (14) into (19), the reliability for detecting user $k$ in MAP sense can be expressed as ${ }^{1}$

$$
L_{k}=4\left(1+\bar{\gamma}_{k}\right)\left|z_{k}\right| \triangleq\left(1+\bar{\gamma}_{k}\right)\left|z_{k}\right|, k=1,2, \ldots, K
$$

Note that, according to the random matrix theory [13], the decision variables $z_{1}, z_{2}, \ldots, z_{K}$ become more independent, satisfying more the condition for (17), as the size $N$ of the DS-CDMA or SDMA systems increases. Hence, the reliability measurement based on (20) converges to the optimum of that based on (16), as the size of the DSCDMA or SDMA systems increases. This in turn implies that even the conventional MMSE-MUD converges (but not fast) to the optimum, as the size of the DS-CDMA or SDMA systems increases.

Upon using the matrix inverse lemma [4] for $\boldsymbol{R}_{k}=\boldsymbol{R}_{y}-\boldsymbol{h}_{k} \boldsymbol{h}_{k}^{H}$, we can obtain

$$
\boldsymbol{R}_{k}^{-1}=\boldsymbol{R}_{y}^{-1}+\frac{\boldsymbol{R}_{y}^{-1} \boldsymbol{h}_{k} \boldsymbol{h}_{k}^{H} \boldsymbol{R}_{y}^{-1}}{1-\boldsymbol{h}_{k}^{H} \boldsymbol{R}_{y}^{-1} \boldsymbol{h}_{k}}
$$

${ }^{1}$ If the source symbols do not satisfy $P\left(b_{k}=+1\right)=P\left(b_{k}=-1\right)$, the reliability in MAP sense can be derived by the first equation in (19), resulting in $L_{k}=\left|4\left(1+\bar{\gamma}_{k}\right) z_{k}+L_{0}\right|, k=1,2, \ldots, K$, where $L_{0}=$ $\ln \left[P\left(b_{k}=+1\right) / P\left(b_{k}=-1\right)\right]$ denotes the a-priori information of $b_{k}$. In this case, (20) is the reliability measured in the sense of ML. 


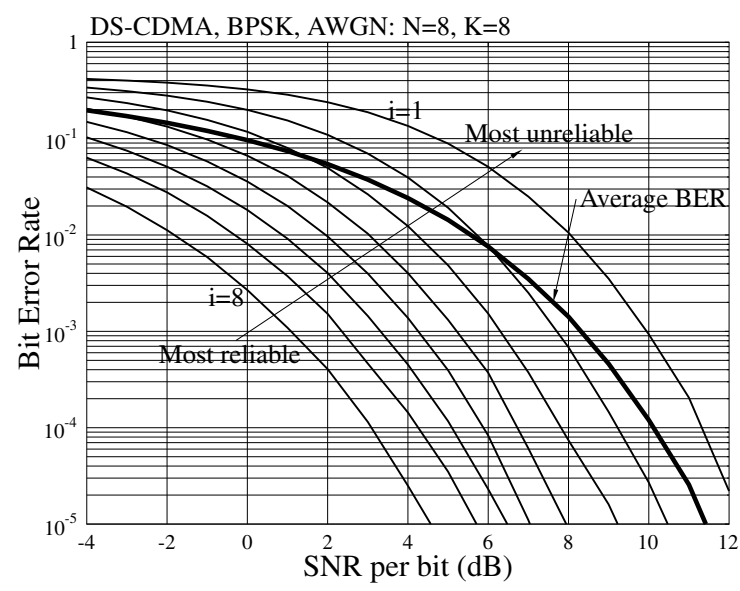

Fig. 3. BER versus SNR per bit $\left(\gamma_{b}\right)$ for the bits at different reliability levels detected by the MMSE-MUD in a DS-CDMA system using BPSK modulation, when communicating over Gaussian channels.

Substituting it into $\bar{\gamma}_{k}=\boldsymbol{h}_{k}^{H} \boldsymbol{R}_{k}^{-1} \boldsymbol{h}_{k}$ and then applying the result to (20), we have

$$
L_{k}=\left(\frac{1}{1-\boldsymbol{h}_{k}^{H} \boldsymbol{R}_{y}^{-1} \boldsymbol{h}_{k}}\right)\left|z_{k}\right|, k=1,2, \ldots, K
$$

The above equation shows that the reliabilities can be directly evaluated based on the auto-correlation matrix $\boldsymbol{R}_{y}$, which in practice can be readily estimated from the observations obtained at receiver.

The ordered BER performance for the DS-CDMA systems employing the MMSE-MUD is shown in Fig. 3. An example showing the ordered BER performance of the SDMA systems in Rayleigh fading channels can be found in Fig. 1 of [10]. Note that, in order to avoid that one user's spreading sequence is the linear combination of some other users' spreading sequences (the probability of this event is very high for $N=8$ ), in our simulations for Fig. 3, we chose a spreading matrix $\boldsymbol{C}$, which yielded a determinant of $\operatorname{det}\left(\boldsymbol{C}^{T} \boldsymbol{C}\right)=0.219727$. Hence, the user sequences used are highly correlated.

Fig. 3 and Fig.1 of [10] shows explicitly that, after the MMSEMUD, the BER of the users at different reliability levels is highly diverse, as that observed in Figs. 1 and 2. Hence, there exists significant RMD in both the DS-CDMA and SDMA systems using MMSEMUD, when communicating over either Gaussian or Rayleigh fading channels.

Note that, when comparing Fig. 3 with Fig. 1, we can see that the ordered BER performance shown in both figures is very similar, except the slight shift on the SNR axis. Hence, we can be implied that the decision variables provided by the MMSE-MUD are highly independent. Furthermore, we can deduce that the SNR shift is the result of the correlation existing among the decision variables.

\section{Receiver Multiuser Diversity Aided Multi-Stage MMSE DETECTION}

From (16) - (18) we can gain insight that, in order for a lowcomplexity IC-based MUD scheme to achieve the close to optimum BER performance, the signals to be detected need to have the following two properties. First, the reliabilities of these signals can be detected in an optimum way near independently. Second, the reliabilities present that some signals are much more reliable than some other signals, so as to be beneficial to use IC. Fortunately, as shown in the last section, the user signals provided by the MMSE-MUD have these properties: the reliabilities can be measured near independently based on (20), and they are highly diverse, generating significant RMD as shown in Figs. 3 and Fig.1 in [10]. Hence, this RMD can be exploited to determine a detection order for the RMD/MS-MMSE MUD. As the detection in V-BLAST systems [7-9], the RMD/MS-MMSE MUD is divided into $K$ detection stages, each stage detects one user that is assumed to be the most reliable of the users having not been detected yet, determined by the reliabilities measured according to (20).

Let $\boldsymbol{R}_{y}^{(0)}=\boldsymbol{R}_{y}$ express the autocorrelation matrix of $\boldsymbol{y}^{(0)}=\boldsymbol{y}$, while $\boldsymbol{R}_{y}^{(s)}$ express the autocorrelation matrix of $\boldsymbol{y}^{(s)}$, which is the output generated by the $s$ th detection stage under the assumption that the previous $(s-1)$ stages of detection are correct. Let the signature of the user detected at the $s$ th stage be denoted by $\boldsymbol{h}^{(s)}$. Furthermore, let after the $s$ th detection stage the signatures of the users having not been detected be collected in $\tilde{\boldsymbol{H}}^{(s)}$, where $\tilde{\boldsymbol{H}}^{(0)}=\boldsymbol{H}$. Then, the detection procedure of RMD/MS-MMSE MUD can be described as follows:

Initialization: $\boldsymbol{y}^{(0)}=\boldsymbol{y}, \boldsymbol{R}_{y}^{(0)}=\boldsymbol{R}_{y}, \tilde{\boldsymbol{H}}^{(0)}=\boldsymbol{H}, \boldsymbol{W}^{(0)}=\boldsymbol{R}_{y}^{-1} \boldsymbol{H}$; Detection: for $s=1,2, \ldots, K$, compute

1) Decision variables: $\boldsymbol{z}^{(s)}=\Re\left\{\left(\boldsymbol{W}^{(s-1)}\right)^{H} \boldsymbol{y}^{(s-1)}\right\}$, where $\boldsymbol{W}^{(s-1)}=\left(\boldsymbol{R}_{y}^{(s-1)}\right)^{-1} \tilde{\boldsymbol{H}}^{(s-1)} ;$

2) Determining the most reliable user: For the users $k_{1}^{\prime}, k_{2}^{\prime}, \ldots$, $k_{K-s+1}^{\prime}$ that have not been detected, compute their reliabilities according to (20), and find the most reliable user of $k^{(s)}=$ $\arg \max _{k_{i}^{\prime}}\left\{L_{k_{1}^{\prime}}, L_{k_{2}^{\prime}}, \ldots, L_{k_{K-s+1}^{\prime}}\right\}$;

3) Decision making: $\hat{b}^{(s)}=\operatorname{sgn}\left(z_{k^{(s)}}^{(s)}\right)$, where $z_{k^{(s)}}^{(s)}$ is the $k^{(s)}$ th entry of $\boldsymbol{z}^{(s)}$;

4) Interference cancellation: $\boldsymbol{y}^{(s)}=\boldsymbol{y}^{(s-1)}-\boldsymbol{h}^{(s)} \hat{b}^{(s)}$;

5) Update: $\boldsymbol{W}^{(s)} \leftarrow \boldsymbol{W}^{(s-1)} ; \quad\left[\tilde{\boldsymbol{H}}^{(s)} \leftarrow\left(\tilde{\boldsymbol{H}}^{(s-1)}, \boldsymbol{h}^{(s)}\right)\right.$, $\left.\boldsymbol{R}_{y}^{(s)}=\boldsymbol{R}_{y}^{(s-1)}-\boldsymbol{h}^{(s)}\left(\boldsymbol{h}^{(s)}\right)^{H}\right]$.

In the above algorithm $\tilde{\boldsymbol{H}}^{(s)}$ is obtained from $\tilde{\boldsymbol{H}}^{(s-1)}$ by deleting the column of $\boldsymbol{h}^{(s)}$ corresponding to the user detected at the $s$ th detection stage. It can be shown that $\tilde{\boldsymbol{H}}^{(s)}=\tilde{\boldsymbol{H}}^{(s-1)} \boldsymbol{P}^{(s)}$, where $\boldsymbol{P}^{(s)}$ is a permutation matrix obtained from $\boldsymbol{I}_{K}$ by removing the $s$ columns corresponding to the users having been detected.

\section{Performance Results}

The BER performance results for the SDMA systems are shown in Fig. 4, while for the DS-CDMA systems are shown in Fig. 5 for Gaussian channels and Fig. 6 for Rayleigh fading channels. In our simulations the DS-CDMA systems were assumed to employ random spreading sequences.

From the BER performance results shown in Figs. 4 - 6, we can have the following observations. First, the RMD/MS-MMSE MUD is a highly efficient MUD scheme that is suitable for diverse communications scenarios. It is highly efficient for both the DS-CDMA and SDMA systems. It is suitable for both Gaussian and fading channels. Furthermore, it can be applied for detection in the multiuser systems that are under-load, full-load or overload. Second, when the DSCDMA or SDMA systems are full-load, i.e., when $K=N$, the RMD/MS-MMSE MUD is generally capable of achieving the nearoptimum BER performance. Third, for all the scenarios considered, at a BER of $10^{-2}$, which is often the BER interested in practical systems before applying error-control decoding, the BER performance of the RMD/MS-MMSE MUD for the DS-CDMA or SDMA systems supporting $K=2 N$ users is much better than that of the MMSEMUD for the DS-CDMA or SDMA systems supporting only $K=N$ users. Finally, at a BER of $10^{-2}$, as shown in Fig. 4 for the SDMA systems and Fig. 5 for the DS-CDMA systems communicating over Gaussian channels, the number of users supported can be doubled from 


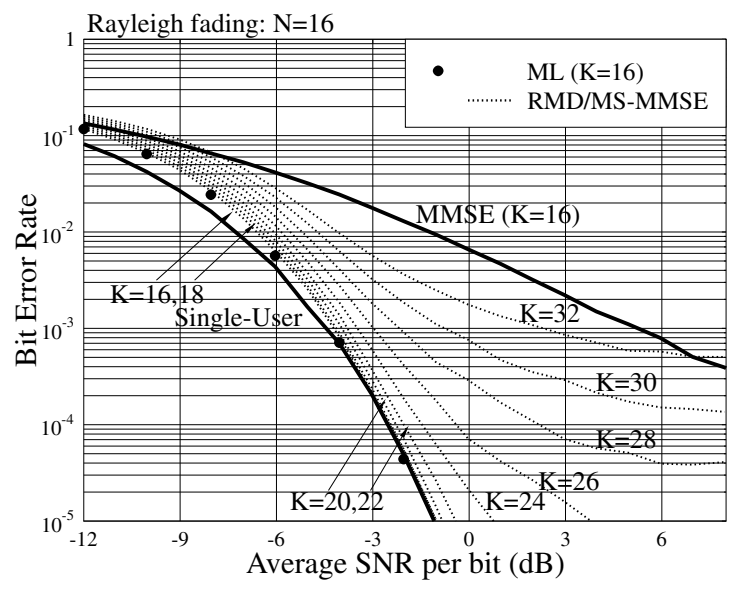

Fig. 4. BER versus average SNR per bit performance of the SDMA systems communicating over Rayleigh fading channels.

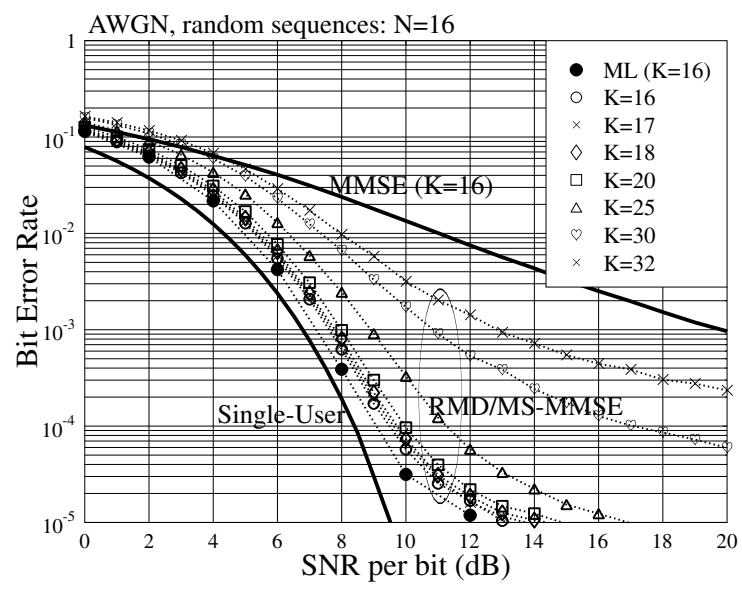

Fig. 5. BER versus average SNR per bit performance of the DS-CDMA systems communicating over AWGN channels.

$K=N$ to $K=2 N$ by increasing the SNR per bit less than $3 \mathrm{~dB}$. When communicating over Rayleigh fading channels, as seen in Fig. 6, the BER performance achieved by the RMD/MS-MMSE MUD for the DS-CDMA systems supporting either $K=N$ or $K=2 N$ users is still similar as the single-user BER bound, provided that the SNR per bit is higher than $13 \mathrm{~dB}$, which results in a BER around $10^{-2}$. Therefore, with the RMD/MS-MMSE MUD, the throughput of DSCDMA or SDMA systems increases at least linearly with the SNR per bit invested.

Note that, in Fig. 5 BER floors are observed for both the ML-MUD $(K=16)$ and our RMD/MS-MMSE MUD. This is because random spreading sequences were assumed, which result in that the probability of two or more users having linearly dependent spreading sequences is higher than $1-\left(1-1 / 2^{N-1}\right)^{K-1}=4.6 \times 10^{-4}$, when $N=16$.

In conclusions, we have shown that the MMSE-MUD for a sufficiently large multiuser system is capable of providing approximately independent detection and generating significant RMD, when measured in the MAP sense. Consequently, the RMD/MS-MMSE MUD designed by making use of these properties is highly efficient for operation in either full-load or overload scenarios, over either Gaussian or fading channels. A full-load DS-CDMA or SDMA system of

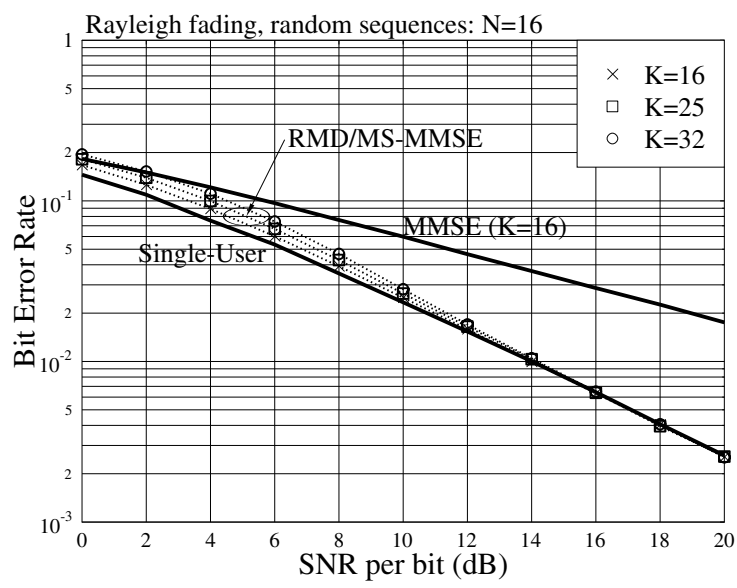

Fig. 6. BER versus average SNR per bit performance of the DS-CDMA systems communicating over flat Rayleigh fading channels.

moderate size is generally capable of achieving the BER performance close to the optimum. Within the BER range of interest, an overload DS-CDMA or SDMA system using the RMD/MS-MMSE MUD to support $K=2 N$ users still significantly outperform a corresponding DS-CDMA or SDMA system using MMSE-MUD to support $K=N$ users. Furthermore, the proposed RMD/MS-MMSE MUD is lowcomplexity, which is at the similar level as a conventional MMSE-SIC or a V-BLAST detector.

\section{REFERENCES}

[1] S. Verdu, "Minimum probability of error for asynchronous multiple access communication systems," in IEEE Military Communications Conference. IEEE, October 1983, pp. 213-219.

[2] — "Minimum probability of error for asynchronous Gaussian multiple-access channels," IEEE Transactions on Information Theory, vol. 32, no. 1, pp. 85 - 96, January 1986.

[3] - Multiuser Detection. Cambridge University Press, 1998

[4] L.-L. Yang, Multicarrier Communications. Chichester, United Kingdom: John Wiley, 2009.

[5] F. Hasegawa and et.al, "Speed and accuracy comparison of techniques for multiuser detection in synchronous CDMA," IEEE Transactions on Communications, vol. 52, no. 4, pp. 540 - 545, April 2004

[6] A. Duel-Hallen, "A family of multiuser decision-feedback detectors for asynchronous code-division multiple-access channels," IEEE Transactions on Communications, vol. 43, no. 2/3/4, pp. 421 - 434, Feb./Mar./Apr. 1995.

[7] P. Wolniansky, G. Foschini, G. Golden, and R. Valenzuela, "V-BLAST: an architecture for realizing very high data rates over the rich-scattering wireless channel," in International Symposium on Signals, Systems, and Electronics (ISSSE). IEEE, 1998, pp. 295-300.

[8] G. Foschini, G. Golden, R. Valenzuela, and P. Wolniansky, "Simplified processing for high spectral efficiency wireless communication employing multi-element arrays," IEEE Journal on Selected Areas in Communications, vol. 17, no. 11, pp. 1841 - 1851, November 1999.

[9] G. Golden, C. Foschini, R. Valenzuela, and P. Wolniansky, "Detection algorithm and initial laboratory results using V-BLAST space-time communication architecture," IEE Electronics Letter, vol. 35, no. 1, pp. 14 16, January 1999.

[10] L.-L. Yang, "Using multi-stage MMSE detection to approach optimum error performance in multiantenna MIMO systems," in IEEE 70th Vehicular Technology Conference: VTC2009-Fall. IEEE, 2023 September 2009.

[11] J. Hagenauer, E. Offer, and L. Papke, "Iterative decoding of binary block and convolutional codes," IEEE Transactions on Information Theory, vol. 42, no. 2, pp. 429-445, March 1996.

[12] H. V. Poor and S. Verdu, "Probability of error in MMSE multiuser detection," IEEE Transactions on Information Theory, vol. 43, no. 3, pp. 858-871, May 1997.

[13] A. M. Tulino and S. Verdu, Random Matrix Theory and Wireless Communications. MA, USA: now Publishers Inc., 2004. 